\title{
Search for Low-mass Dark-Sector Gauge Boson with the BABAR Detector
}

\author{
Romulus Godang* \\ On Behalf of the BABAR Collaboration \\ Department of Physics \\ University of South Alabama \\ 411 University Boulevard, North \\ Mobile, AL 36688 \\ E-mail: godangesouthalabama.edu
}

We report searches for a new muonic dark force mediated by a gauge boson $\left(Z^{\prime}\right)$ coupling only to the second and third lepton families. The existence of the $Z^{\prime}$ boson is probed in $e^{+} e^{-} \rightarrow \mu^{+} \mu^{-} Z^{\prime}$, $Z^{\prime} \rightarrow \mu^{+} \mu^{-}$events based on the full data sample collected with the BABAR detector at the PEPII $e^{+} e^{-}$collider. No significant signal is observed. Limits on dark-sector coupling constants are derived; these improve upon current bounds, and further constrain the allowed parameter space.

$38^{\text {th }}$ International Conference on High Energy Physics

3-10 August 2016

Chicago, USA

\footnotetext{
${ }^{*}$ Speaker.

${ }^{\dagger}$ This work was supported by the U.S. Department of Energy
} 


\section{Introduction}

Some models of physics beyond Standard Model (SM) predict the existence of a new nonAbelian gauge group Higgs with gauge boson masses below $10 \mathrm{GeV}$ [1]. The WIMP hypothesis suggested that dark matter is assumed to consist of stable particle with low masses. Such new gauge bosons can typically interact with other Standard Model elementary particles. Based on the $L_{\mu}-L_{\tau}$ model [2] the most promising candidate is the gauge group associated with the difference between muon and tau-lepton number. The gauge $L_{\mu}-L_{\tau}$ predicts that the $Z^{\prime}$ is being coupled only to the second and third generation of leptons.

\section{The BABAR Detector and Data Set}

We used the data collected by the BABAR detector with the total luminosity of $514 \mathrm{fb}^{-1}$. A detail description of the BABAR detector is presented elsewhere [3]. The data were taken at the $\Upsilon(4 S)$ resonance plus $28 \mathrm{fb}^{-1}$ taken at $\Upsilon(3 S), 14 \mathrm{fb}^{-1}$ taken at $\Upsilon(2 S)$, and $48 \mathrm{fb}^{-1}$ taken at $40 \mathrm{MeV}$ below the $\Upsilon(4 S)$ (off-resonance). The $\Upsilon(4 S)$ resonance decays to a pair of $\bar{B} B$ [4]. The data was only examined after finish finalizing the analysis method. For the background study we generated signal Monte Carlo (MC) samples. Signal MC events are generated using MadGraph 5 [5], which calculates matrix elements for the sample. The MC then were showered using Pythia 6 [6] for about 30 different $Z^{\prime}$ mass hypotheses. The most dominant background comes from the QED processes. We generate the direct processes of $e^{+} e^{-} \rightarrow \mu^{+} \mu^{-} \mu^{+} \mu^{-}$using Diag36 [7], which includes the full set of the lowest order diagrams. The events of the process of $e^{+} e^{-} \rightarrow e^{+} e^{-}(\gamma)$ is generated using BHWIDE [8] and the MC events of $e^{+} e^{-} \rightarrow \mu^{+} \mu^{-}(\gamma)$ and $e^{+} e^{-} \rightarrow \tau^{+} \tau^{-}(\gamma)$ are generated using KK [9]. The off-resonance data samples, $e^{+} e^{-} \rightarrow \bar{q} q(\mathrm{q}=\mathrm{u}, \mathrm{d}, \mathrm{s}, \mathrm{c})$, are simulated using EvtGen [10]. The detector acceptance and reconstruction efficiency are determined using MC simulation based on GEANT4 [11].

\section{Measurement of $Z^{\prime} \rightarrow \mu^{+} \mu^{-}$}

Events with the process of $e^{+} e^{-} \rightarrow \mu^{+} \mu^{-} Z^{\prime}$ and $Z^{\prime} \rightarrow \mu^{+} \mu^{-}$final state that exactly contain two pairs of oppositely charged tracks are selected. The muons are identified by particle identification algorithms for each track. We require the sum of energies of the electromagnetic clusters that are not associated to any track must be less than $200 \mathrm{MeV}$. We finally reject events that come from the $\Upsilon(3 S)$ and $\Upsilon(2 S)$, where $\Upsilon(2 S, 3 S) \rightarrow \pi^{+} \pi^{-} \Upsilon(1 S), \Upsilon(1 S) \rightarrow \mu^{+} \mu^{-}$decays if the dimuon combination is within $100 \mathrm{MeV}$ of the $\Upsilon(1 S)$.

Figure 1 (left) shows the reduced dimuon mass which is calculated by $m_{R}=\sqrt{m_{\mu^{+} \mu^{-}}^{2}-4 m_{\mu}^{2}}$ in a log scale. The most dominant samples is coming from the direct decay of $e^{+} e^{-} \rightarrow \mu^{+} \mu^{-} \mu^{+} \mu^{-}$ process. The contribution from the decay of $\Upsilon(2 S) \rightarrow \pi^{+} \pi^{-} J / \psi, J / \psi \rightarrow \mu^{+} \mu^{-}$is shown around $3 \mathrm{GeV}$. The signal yield is extracted from the unbinned likelihood fits to the reduced dimuon mass spectrum within the range of $0.212<m_{R}<10 \mathrm{GeV}$ for the $\Upsilon(4 S)$ resonance data and $0.212<m_{R}<$ $9 \mathrm{GeV}$ for $\Upsilon(2 S)$ and $\Upsilon(3 S)$ data. A region of $\pm 30 \mathrm{MeV}$ around the nominal known $J / \psi$ mass is excluded. The total of 2219 mass hypotheses are found. The cross section of $e^{+} e^{-} \rightarrow \mu^{+} \mu^{-} Z^{\prime}$, $Z^{\prime} \rightarrow \mu^{+} \mu^{-}$is extracted as a function of $Z^{\prime}$ mass as shown in Fig. 1 (right). 

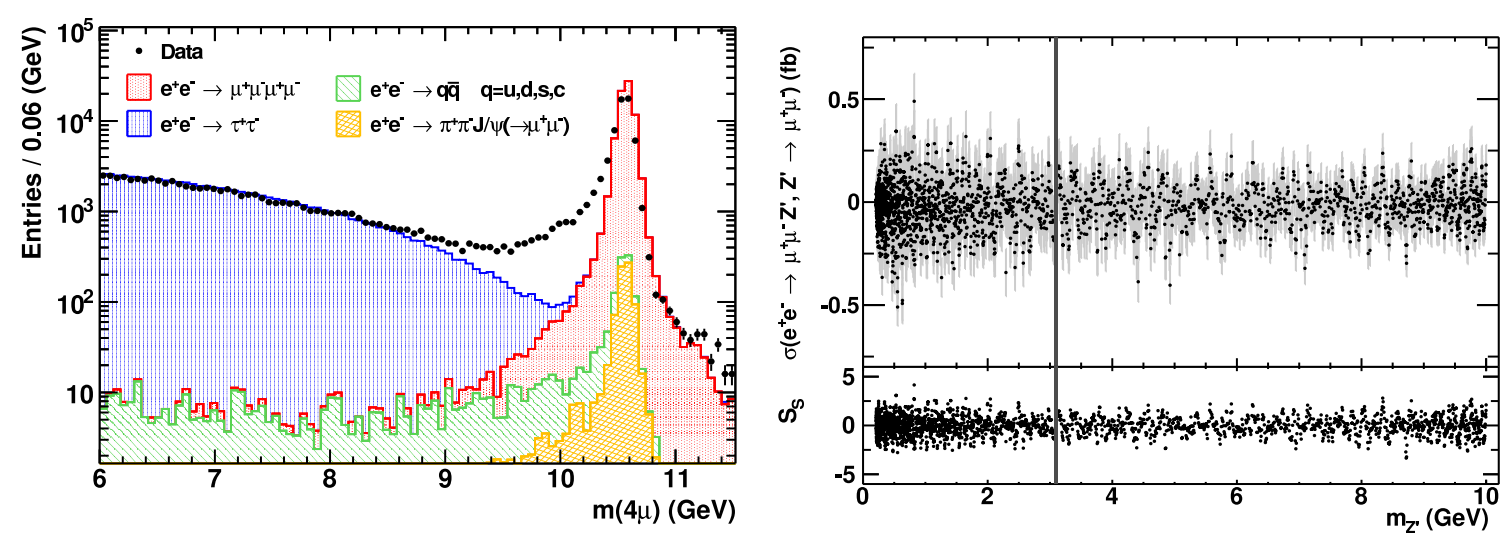

Figure 1: Left: The distribution of the reduced dimuon mass, $m_{R}=\sqrt{m_{\mu^{+} \mu^{-}}^{2}-4 m_{\mu}^{2}}$ in a $\log$ scale with the various processes of the Monte Carlo events. It is normalized to the data luminosity. Right: The measurement of $e^{+} e^{-} \rightarrow \mu^{+} \mu^{-} Z^{\prime}, Z^{\prime} \rightarrow \mu^{+} \mu^{-}$cross section as a function of the $Z^{\prime}$ mass. The excluded region is indicated by the gray band.

We exclude the $J / \psi$ region when calculating the correction factors by fitting the simulated and reconstructed reduced dimuon masses in the range of $1<m_{R}<9 \mathrm{GeV}$. The cross section of $e^{+} e^{-} \rightarrow$ $\mu^{+} \mu^{-} Z^{\prime}, Z^{\prime} \rightarrow \mu^{+} \mu^{-}$is extracted as a function of $Z^{\prime}$ mass as shown in Fig. 2 (left). The gray band indicates the excluded region. We find the largest local significance is $4.3 \sigma$ around $Z^{\prime}$ mass of $0.82 \mathrm{GeV}$ that is corresponding to the global significance of $1.6 \sigma$. It is consistent with the zero-hypothesis. We derive $90 \%$ confidence level (CL) Bayesian upper limit on the cross section of $e^{+} e^{-} \rightarrow \mu^{+} \mu^{-} Z^{\prime}, Z^{\prime} \rightarrow \mu^{+} \mu^{-}$. We finally extract the corresponding $90 \% \mathrm{CL}$ on the coupling parameter $g^{\prime}$ by assuming the equal magnitude vector couplings muons, taus, and the corresponding neutrinos together with the existing limits from Borexino and neutrino experiments as shown in Fig. 2 (right).
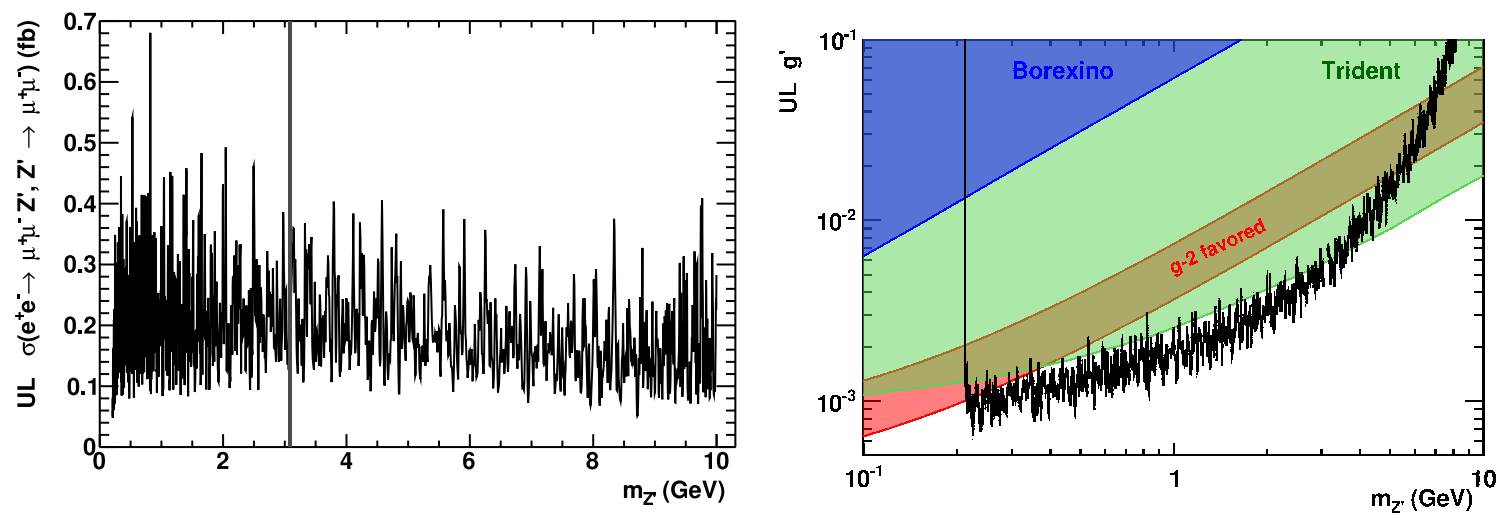

Figure 2: Left: The limit on the cross section $\sigma\left(e^{+} e^{-} \rightarrow \mu^{+} \mu^{-} Z^{\prime}, Z^{\prime} \rightarrow \mu^{+} \mu^{-}\right)$as a function of the $Z^{\prime}$ mass. The excluded region is indicated by the gray band. Right: Upper limit on the new gauge coupling $g^{\prime}$ as a function of the mass of $Z^{\prime}$ together with the existing limits from Borexino and neutrino experiments. 


\section{Summary}

In summary, we have performed the first direct measurement of $Z^{\prime}$ production from the decay of $e^{+} e^{-} \rightarrow \mu^{+} \mu^{-} Z^{\prime}, Z^{\prime} \rightarrow \mu^{+} \mu^{-}$at BABAR. No significant signal is observed for $Z^{\prime}$ masses in the range of $0.212-10 \mathrm{GeV}$. We set limits on the coupling parameters $g^{\prime}$ down to $7 \times 10^{-4}$ near the dimuon threshold. We set a strongest bounds for much of the parameter space below $3 \mathrm{GeV}$. We exclude most of the remaining parameter space preferred by the discrepancy between the calculated and measured anomalous magnetic moment of the muon above the dimuon threshold [12].

\section{Acknowledgments}

The author would like to thank B. Echenard for his helpful suggestions. The author also thank the organizers of the $38^{\text {th }}$ International Conference on High Energy Physics, the BABAR Collaboration, the University of South Alabama, and the University of Mississippi.

\section{References}

[1] A. Birkedal, K. Matchev, and M. Perelstein, Phys. Rev. D 70, 077701 (2004)

[2] W. Altmannshofer, S. Gori, M. Pospelov, and I. Yavin Phys. Rev. D 89, 095033 (2014)

[3] B. Aubert et al., (BABAR Collaboration), Nucl. Instrum. Meth. A 479, 1 (2002);

B. Aubert et al., (BABAR Collaboration), Nucl. Instrum. Meth. A 729, 1 (2013)

[4] B. Aubert et al., (BABAR Collaboration), Phys. Rev. Lett. 95, 042001 (2005)

[5] J. Alwall et al., JHEP 1407, 079 (2014)

[6] T. Sjostrand, S. Mrenna, and P. Z. Skands, JHEP 0605, 026 (2006)

[7] F. A. Berends, P. H. Daverveld and R. Kleiss, Nucl. Phys. B 253, 441 (1985)

[8] S. Jadach, W. Placzek, and B. F. L. Ward, Phys. Lett. B 390, 298 (1997)

[9] S. Jadach, B. F. L. Ward, and Z. Was, Phys. Rev. D 63, 113009 (2001)

[10] D. J. Lange, Nucl. Instrum. Meth. A 462, 152 (2001)

[11] S. Agostinelli et al., (GEANT4 Collaboration), Nucl. Instrum. Methods Phys. Res., Sect. A 506, 250 (2003)

[12] J. P. Lees et al., (BABAR Collaboration), Phys. Rev. D 94, 011102 (2016) 